\title{
Role of Haptic Interfaces in Robot-Assisted Minimally Invasive Surgery
}

\section{Tauseef Gulrez*}

Virtual and Interactive Simulations of Reality Laboratory, Department of Computing, Macquarie University, Sydney, Australia

\section{Editorial}

Robot-Assisted Minimally Invasive Surgery (RAMIS) was introduced in the mid-eighties, when a PUMA robotic arm was used in a neurosurgical biopsy, successfully. RAMIS has evolved since then and has brought a revolution in surgical procedures. In 1990 the AESOP system was introduced by Computer Motion, became the first robotic endoscopic surgical system. Ten years later in year 2000 a fullfledged master/slave Da Vinci robotic surgical system was introduced by Intuitive Surgical shown in (Figure 1), consists of a master console and a slave robotic system. The surgeon sits in-front of the master control system and controls the robotic manipulators at the slave end through a stereoscopic $3 \mathrm{D}$ view projecting inners of the patient's body. The robotic manipulators and a camera, penetrate inside the patient's body through small incisions. This technology has reduced not only post-operation recovery time, but also less risk of infection and surgical trauma. Although, there are numerous advantages of RAMIS which can enhance the dexterity and precision of surgeons. However, presently available RAMIS systems do not convey force information at the manipulator's end in the form of haptic feedback to the surgeon.

The loss of haptic feedback to the surgeon, can cause tissue or suture damage during robotic surgery, due to the excessive force applied at the manipulator's end especially during knot- tying process. Therefore, there is a need to incorporate haptic feedback mechanism in surgical robotic master interfacing systems and it is proven that lack of haptic feedback played a role in this problem. Haptic is a broad term, defining kinesthetic (force) and cutaneous (tactile) feedback.

In this editorial, we will focus on the challenges faced in designing tactile feedback interface systems for RAMIS. Majority of tactile feedback systems have been designed for mounting on surgical instruments themselves. However, there is still a gap to be filled where tactile system is intended to use with existing RAMIS systems. The tactile feedback technology also requires that sensors to be embedded at the gripper-end in RAMIS environment within the patient's body and the acquired interaction to be translated into tactile display to the surgeon.

Haptic systems which provide tactile feedback to human skin are usually referred to as cutaneous feedback systems. In RAMIS, the concept of providing cutaneous feed- back on surgeon's fingers as a function of force, pressure, temperature, texture, etc. without sacrificing dexterity and movement is a technical challenge and is considered as a significant limitation of a RAMIS.

Presently, different techniques and devices have been introduced as tactile user interfaces, e.g. arrays of capacitive and force-sensitive resistors [1] instrumented membranes [2] and micro machined piezoelectric arrays [3]. These tactile display technologies create a perception that surgeon's fingertip is directly in contact with the patient's organ or surgical component such as suture. Most commonly used user interfaces displaying the tactile information are made up of array of pins which individually actuate mapping the data to their position commands.

Another major issue in designing of tactile user interfaces is the bilateral tele-operation. There has been an abundance of research

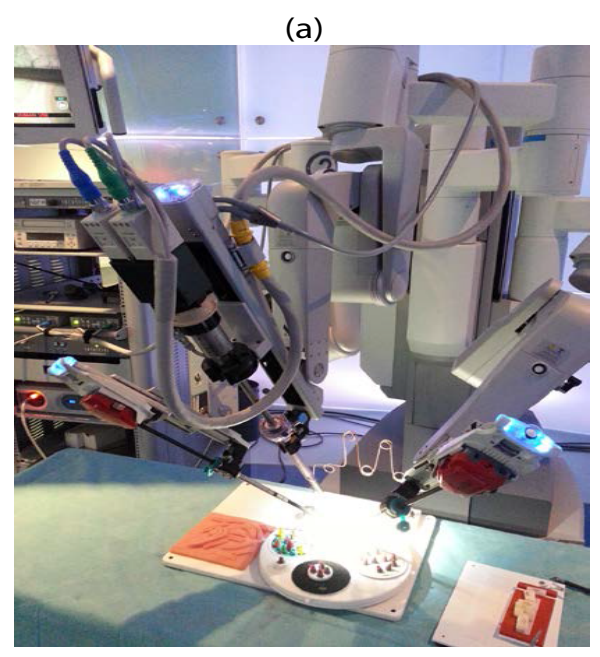

(b)

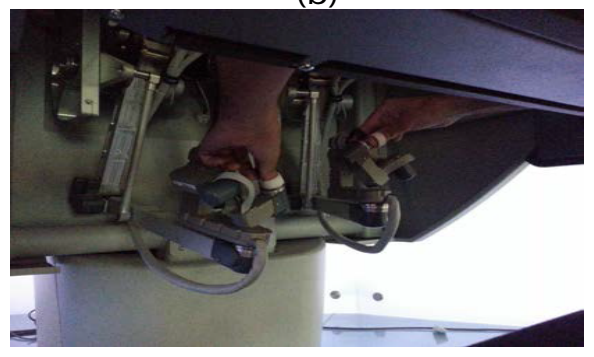

Figure 1: Da Vinci system - (a) The slave surgical robotic system. (b) Da Vinci user-interface, master control interface (without haptic feedback) for surgeon.

on bilateral tele-operation control [4] although fewer contributions address surgical robotics. Just as is the case for non-surgical systems $[5,6]$ when designing controllers for surgical purpose the stabilitytransparency trade-off must be faced. Since making compromises on system stability is hardly an option, the transparency that can be reached will always be somewhat limited in surgical applications. One way to balance this trade-off towards improved performance exists in exploiting the knowledge about the dynamics of operator and surgical environment that the system will interact with. Compared to passivity-based schemes [7] controllers that exploit the fact that neither patients nor surgeons are infinitely stiff improve performance

*Corresponding author: Tauseef Gulrez, Virtual and Interactive Simulations of Reality Laboratory, Department of Computing, Macquarie University, Sydney, NSW 2109, Australia, Tel: 0044764 932; E-mail: gtauseef@ieee.org

Received November 11, 2013; Accepted November 13, 2014; Published November 28, 2014

Citation: Gulrez T (2014) Role of Haptic Interfaces in Robot-Assisted Minimally Invasive Surgery. Int J Swarm Intel Evol Comput 4: e107. doi: 10.4172/20904908.1000e107

Copyright: (c) 2014 Gulrez T. This is an open-access article distributed under the terms of the Creative Commons Attribution License, which permits unrestricted use, distribution, and reproduction in any medium, provided the original author and source are credited. 
Citation: Gulrez T (2014) Role of Haptic Interfaces in Robot-Assisted Minimally Invasive Surgery. Int J Swarm Intel Evol Comput 4: e107. doi: 10.4172/20904908.1000e107

Page 2 of 2

without giving in on stability. Nevertheless, since practical systems (such as a da Vinci Surgical System) have many degrees of freedom and possess rather flexible links, the kinematic map of such manipulator changes depending on the load. This negatively affects the achievable transparency. Therefore, these days a lot of attention is required towards bilateral control for tactile user interfaces for surgical robots.

\section{References}

1. Schostek S, Ho CN, Kalanovic D, Schurr MO (2006) Artificial tactile sensing in minimally invasive surgery - a new technical approach. Minim Invasive Ther Allied Technol 15: 296-304.

2. Dargahi J, Najarian S (2004) A supported membrane type sensor for medical tactile mapping. Sensor Review 24: 284-297.
3. Dargahi J, Parameswaran M, Payandeh S (2000) A micromachined piezoelectric tactile sensor for an endoscopic grasper-theory, fabrication and experiments. Journal of Microelectromechanical Systems 9: 329-335.

4. Hokayem PF, Spong MW (2006) Bilateral teleoperation: An historical survey. Automatica 42: 2035-2057.

5. Gulrez T, Tognetti A (2014) A sensorized garment controlled virtual robotic wheelchair. Journal of Intelligent \& Robotic Systems 74: 847-868.

6. Gulrez T (2014) Learnable robotic interfaces. International Journal of Swarm Intelligence and Evolutionary Computation 3:1.

7. Anderson RJ, Spong MW (1989) Bilateral control of tele-operators with time delay Automatic Control. IEEE Transactions on 34: 494-501. 